\title{
LIMB SALVAGE AND MAJOR RECONSTRUCTION SURGERY FOR PATIENTS WITH MALIGNANT BONE TUMORS
}

doi: 10.2478/rojost-2018-0035

Ș. Dragosloveanu $u^{1,2}$, C. Dragosloveanu ${ }^{1}$, D. Cotor ${ }^{1}$, B. Crețu, $u^{1,2}, C l$. Stoica ${ }^{1,2}$

1"Foişor" Orthopaedics-Traumatology and OsteoarticularTB Hospital, Bucharest, Romania

2"Carol Davila" University of Medicine and Pharmacy, Bucharest, Romania

Introduction. Osteosarcoma and chondrosarcoma are the most common bone neoplasms.

The most frequent localization of the osteosarcomas is the lower extremity around the knee and the pelvis and proximal femur for chondrosarcomas. In such cases, the amputation is a common procedure but the limb salvage surgery has become more popular due to the development of chemotherapy and improved diagnostic techniques.

Methods. In the present study, the authors performed a retrospective study of patients diagnosed with osteosarcoma and chondrosarcoma around the knee and hip joint, treated with resection and reconstruction surgery. We evaluated the patients preoperative and postoperative using MRI and scintigraphy. The preoperative planning was made using Cedara I-View. Follow-up was between 1 and 4 years.

Patients. We included 2 patients with chondrosarcoma and 4 patients with other types of sarcomas. All tumors were localized in the lower limb area except for one case, which presented a fast growing fibroblastic sarcoma in the supraspinatus fossa. Using MRI, the tumors were staged Enneking Ila and IIb. Patients with tumor infiltration of nerves or vessels, massive soft tissue infiltration, or pathologic bone fractures were excluded from our study. All the cases included were diagnosed based on incisional biopsy.

Results. We recorded difficulties encountered in resection of the tumor, matching the preoperative planning with the intraoperative findings, rate of recurrence and soft tissue management. Our paper followed our results using GMRS type implants or straight reconstruction in the upper limb. Only one patient presented with lumbar metastatic disease after osteosarcoma after 2 years.

Conclusion. Reconstructive surgery seems a good choice for careful selected patients. This type of surgery is demanding and experience is needed.

We believe that a longer follow-up is needed for young patients to better evaluate the implant stability for mid-to long term.

Keywords: malignant bone tumors, limb salvage surgery, reconstruction surgery, osteosarcoma, chondrosarcoma 